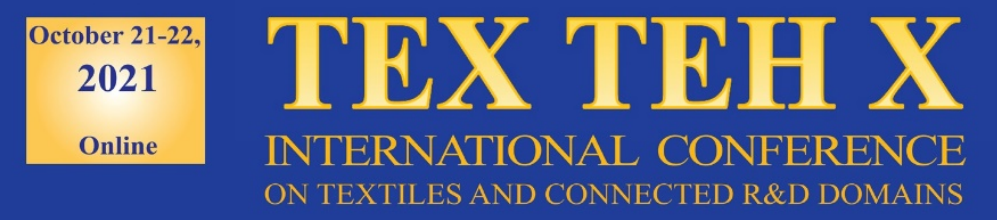

\title{
WATER REPELLENT BREATHABLE PET/WOOL FABRIC VIA PLASMA POLYMERISATION TECHNOLOGY
}

DOI: 10.35530/TT.2021.50

\author{
A. Haji ${ }^{1 *}$, M. Khajeh Mehrizi ${ }^{1}$, M. Ali Tavanai ${ }^{2}$, M. Gohari ${ }^{1}$ \\ ${ }^{1}$ Textile Engineering Department, Yazd University, Yazd, Iran \\ (E-mail: ahaji@yazd.ac.ir,mkhajeh@yazd.ac.ir, mgrock72@yahoo.com) \\ ${ }^{2}$ Textile Engineering Department, Amirkabir University of Technology, Tehran, Iran \\ (E-mail: tavanaie@aut.ac.ir)
}

\begin{abstract}
Water-repellent textiles are usually prepared by application of hydrophobic polymers such as fluorocarbons on fabrics using padding or spraying methods followed by drying and curing steps. These procedures impart hydrophobicity to the fabric, but harm the physical and handle properties of the fabric. In this study, low-pressure plasma was employed for the polymerization of $1 \mathrm{H}, 1 \mathrm{H}, 2 \mathrm{H}, 2 \mathrm{H}$-Perfluorooctyl acrylate on PET/Wool fabric for obtaining water-repellent properties with minimum effect on other desirable properties. To compare the results with the conventional industrial processes, a sample was treated with a commercial water-repellent agent using pad-dry-cure method. The water contact angle, bending length, tensile strength, air permeability, and surface morphology of the samples were compared. The plasma-treated sample showed similar water contact angle and higher fastness properties compared with the sample prepared by the conventional method. The tensile strength of the samples was similar, while the air permeability of the plasmatreated sample was higher and the coating was more uniform compared with the sample prepared by the paddry-cure method.
\end{abstract}

Keywords: fluorocarbon, plasma, polyester, water repellent, wool

\section{INTRODUCTION}

Water-repellent textiles are highly demanded especially for outdoor use, such as tents and outdoor clothing. Silicon or fluorine compounds are usually employed for hydrophobic finishing of textiles and are usually applied by padding, coating or spraying processes. It is important that the fabric retain its physical properties such as tensile strength, tearing strength, handle, and air permeability after being treated with a water repellent finishing agent $[1,2]$.

Pad-dry-cure is the most commonly used method for water-repellent finishing of textiles. The application of high amounts of water repellent agent at elevated temperature usually causes severe damage on the textile fibres. It also consumes a lot of energy and is not environmentally-friendly. Plasma technology is one of the alternative methods for application and polymerization of water repellent monomers on textiles with minimum effect on their physical properties. Plasma treatment also improves the adhesion between the water repellent film and the textile fibres, which improves the fastness properties of the finished goods [3,4].

Plasma treatment can be employed in water repellent finishing of textiles in two mays. Pre-treatment of the textile surface with oxygen, air, or nitrogen plasma can enhance the adhesion of conventional water repellent finishing agents on the fibres. Also, it is possible to polymerise and graft some gases such as $\mathrm{CF}_{4}, \mathrm{C}_{2} \mathrm{~F}_{6}, \mathrm{SF}_{6}, \mathrm{SiH}_{4}$, etc. on textile fibres using 
plasma. In this case, the plasma discharge must occur in the fluorine or silicone containing gas $[3,5,6]$. By the aim of plasma enhanced chemical vapor deposition method, thin inert fluorocarbon coatings can be deposited uniformly on the surface of any type of textile material in a single stage process with the minimum use of chemicals, water, and energy. In this process, the composition, uniformity, morphology, and thickness of the coating can be easily controlled and water repellent, oil repellent, and anti-fouling properties can be obtained by the proper choose of the precursors [7].

Zanini et al. employed an atmospheric pressure plasma pre-treatment to improve the hydro- and oleo-repellent finishing of cashmere and wool/nylon fabrics coated with a commercial fluorocarbon resin by a pad-dry process. Dielectric barrier discharge plasma treatment using air/water vapour mixture as the processing gas improved the uniformity of coverage and enhanced the hydro- and the oleo-repellent properties of the finished fabrics [8].

Ramamoorthy et al. grafted a $\mathrm{C}_{6}$ fluorocarbon (2-(perfluorohexyl) ethyl acrylate) on cotton fabric using atmospheric pressure plasma. The monomer was evaporated and injected in the glow discharge region of the plasma treatment reactor. Argon was used as the carrier gas. Cotton fabric was slowly moved through this region and a thin film of the fluro-polymer was deposited on the surface of the cotton fibres. The resulting fabric showed water, alcohol, and oil repellent properties comparable with the samples prepared by the conventional paddry-cure process. The water repellent property was durable against soxhlet extraction, which confirms the good adhesion between the coating and cotton fibres [9].

In this study, PET/Wool fabric was treated with a commercial water repellent agent by a pad-dry-cure process and compared with another sample prepared by direct polymerization of $1 \mathrm{H}, 1 \mathrm{H}, 2 \mathrm{H}, 2 \mathrm{H}$-Perfluorooctyl acrylate using low-pressure plasma.

\section{EXPERIMENTAL}

\subsection{Materials}

1H,1H,2H,2H-Perfluorooctyl acrylate (figure 1) was purchased from Sigma-Aldrich (USA). Repellan EPF (Fluorocarbon based water repellent agent) was obtained from Pulcra chemicals GmbH (Germany). Twill weave 55\% polyester/45\% wool fabric with a weight of $237 \mathrm{~g} / \mathrm{m}^{2}$ and $\mathrm{Nm}$ (yarn count) $=44$ (two ply) was obtained from Motahari spinning and weaving company, Qazvin, Iran. Non-ionic detergent (Ultravon GPN) was obtained from Huntsman (Switzerland).

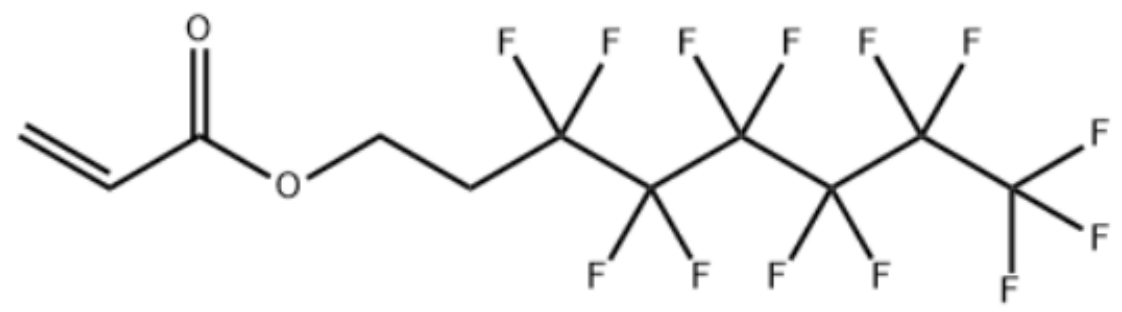

Figure 1. The chemical structure of $1 \mathrm{H}, 1 \mathrm{H}, 2 \mathrm{H}, 2 \mathrm{H}$-Perfluorooctyl acrylate

\subsection{Methods}

To remove any impurities, the fabric samples were scoured using a solution containing $2 \mathrm{~g} / \mathrm{l}$ non-ionic detergent and $2 \mathrm{ml} / \mathrm{l}$ ammonia ( $\mathrm{L}: \mathrm{G}=30: 1)$ at $60^{\circ} \mathrm{C}$ for $45 \mathrm{~min}$, then rinsed with distilled water and dried at ambient temperature.

Plasma treatment was done using a low-pressure equipment made by BasaFan company, Iran (Plasma DEJ BF60). Before introduction of the vapour of the fluorocarbon monomer to the plasma treatment chamber, the surface of the fabric was activated by 
oxygen/argon plasma for 2 minutes at power of $100 \mathrm{~W}$. The flow rate of oxygen and argon was $50 \mathrm{Sccm} .1 \mathrm{H}, 1 \mathrm{H}, 2 \mathrm{H}, 2 \mathrm{H}$-Perfluorooctyl acrylate was heated in a bottle to $50^{\circ} \mathrm{C}$ and the vapour was introduced to the chamber with a flow rate of $300 \mathrm{Sccm}$. Argon gas with a flow rate of $20 \mathrm{Sccm}$ was also used as the carrier gas. Pasma was ignited for 10 minutes at power of $100 \mathrm{~W}$. Finally, the sample was removed and used for further characterizations.

For commercial water repellent finishing, the fabric was padded with $40 \mathrm{~g} / \mathrm{l}$ Repellan $\mathrm{EPF}$ at $\mathrm{pH}=5.5$. The pick-up was $60 \%$. The sample was dried at $110^{\circ} \mathrm{C}$ and cured at $150^{\circ} \mathrm{C}$ for 2 minutes.

The surface morphology of the samples was investigated using SEM images taken on a Vega3 scanning electron microscope (Tescan, Czech Republic). FTIR spectra was obtained using a Vertex 70 (Brucker, Germany) spectrometer in the range of $400-4000 \mathrm{~cm}^{-1}$. The bending length, air permeability, and tensile strength of the samples were measured according to BS-3356, BS-5636, and ASTM D5035 standards, respectively. Washing fastness was evaluated according to the ISO 105-C03: 1994 standard. The contact angle of the fabrics was measured according to the method described in the literature $[10,11]$.

\section{RESULTS AND DISCUSSION}

\subsection{Contact angle}

Table 1 shows the contact angle (CA) of water droplets on raw fabric as well as the samples prepared by plasma treatment and commercial method at time of zero and after 30 seconds. The samples have been washed according to ISO 105-C03: 1994 standard and the CA of the washed samples is reported as well. It can be seen that the raw sample has the lowest contact angle at the beginning and it is decreased drastically after $30 \mathrm{~s}$. The contact angle of water droplet on the plasma-treated sample is much higher than the raw sample and lower than the commercially prepared sample at time of zero and after $30 \mathrm{~s}$. But comparing the contact angles of the washed samples, the plasma-treated sample showed a higher contact angle even after $30 \mathrm{~s}$ of contact with the fabric. This confirms the better fastness of the water repellence imparted to the fabric by plasma polymerization. It is due to the grafting of the water repellent film to the surface of the fibres and higher crosslinking of the plasma polymerized film.

Table 1. Contact angles of the samples prepared by plasma and commercial methods

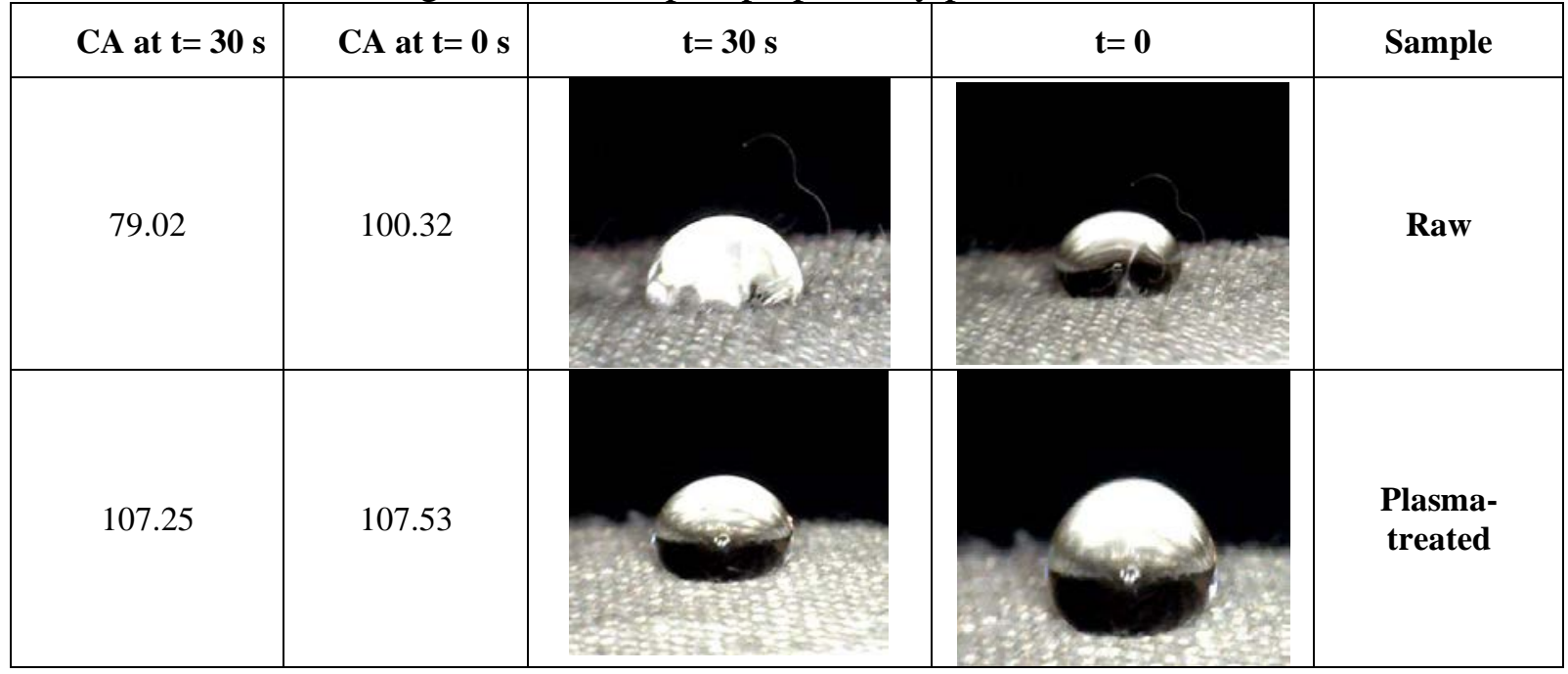




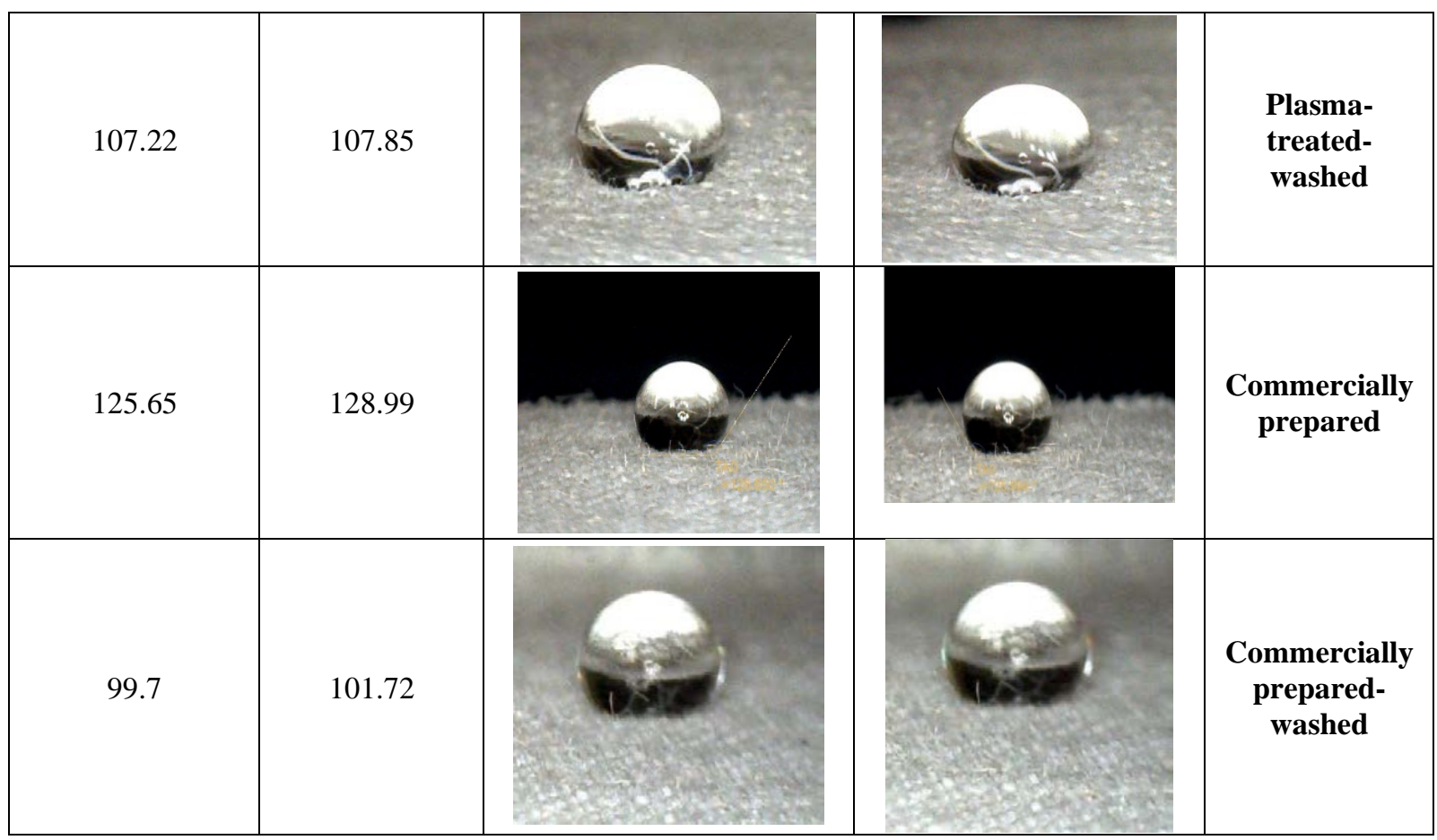

\subsection{Surface morphology}

Figure 2 shows the SEM images of raw fabric as well as the samples prepared by plasma treatment and commercial method. It can be seen that the fibres have been coated more uniformly using the plasma polymerization compared with the conventional pad-dry-cure method.
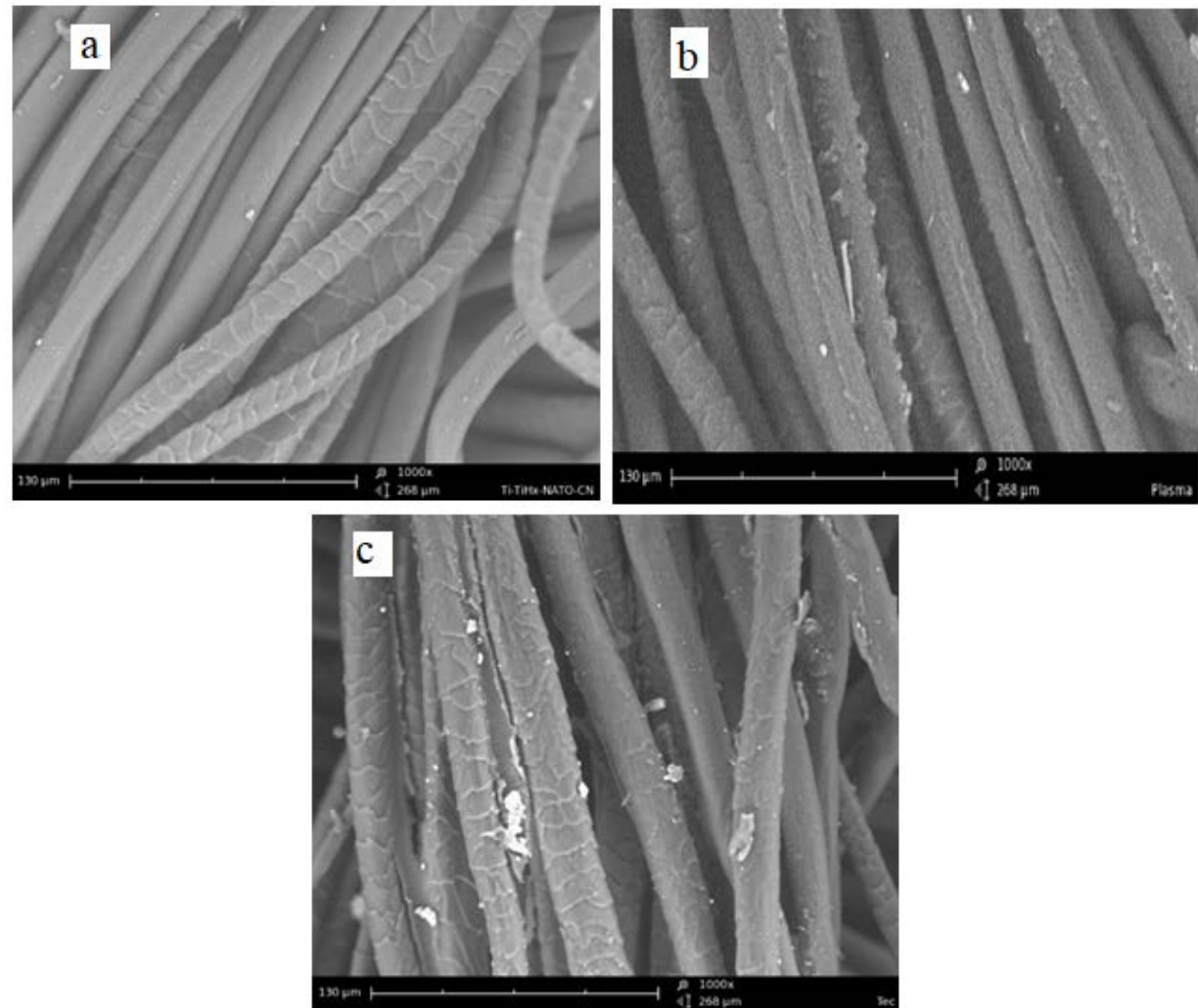

Figure 2. SEM images of: a - raw; b - plasma-treated; c - commercially treated samples 
Also, it can be seen that the spaces between the fibres have been filled with the water repellent resin and the fibres have been sticked together which can affect the handle and breathability of the fabric.

\subsection{FTIR spectroscopy}

The FTIR spectra of the raw, plasma-treated and commercially treated samples are shown in figure 3. The FTIR spectra shows no significant difference between the raw and plasma-treated samples, which may be due to the thinness of the coating. The peak at 1620 $\mathrm{cm}^{-1}$ which corresponds to the carbonyl groups of wool and PET fibres has been diminished in the spectrum of the commercially-treated sample which means the coating of the surface of the fibres with a comparably thick layer of the hydrophobic coating. Figure 4 shows a schematic presentation of the grafting and polymerization of $1 \mathrm{H}, 1 \mathrm{H}, 2 \mathrm{H}, 2 \mathrm{H}$-Perfluorooctyl acrylate on wool and PET fibres in the presence of plasma. Ar/ $\mathrm{O}_{2}$ plasma etches and activates the surface and subsequently a thin film of crosslinked fluoropolymer is grafted on the activated fibres in the next stage.

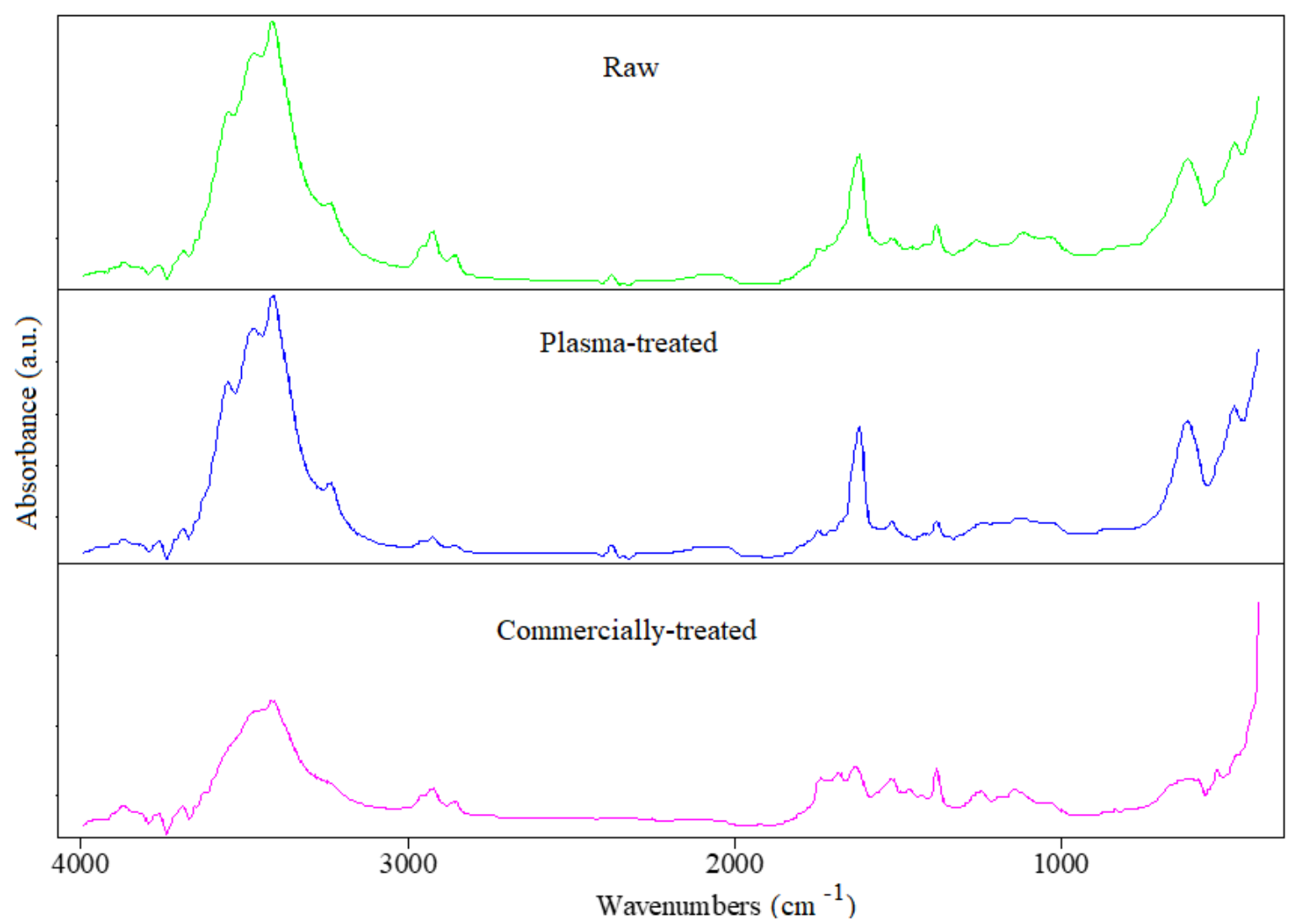

Figure 3. FTIR spectra of raw, plasma-treated, and commercially-treated samples 


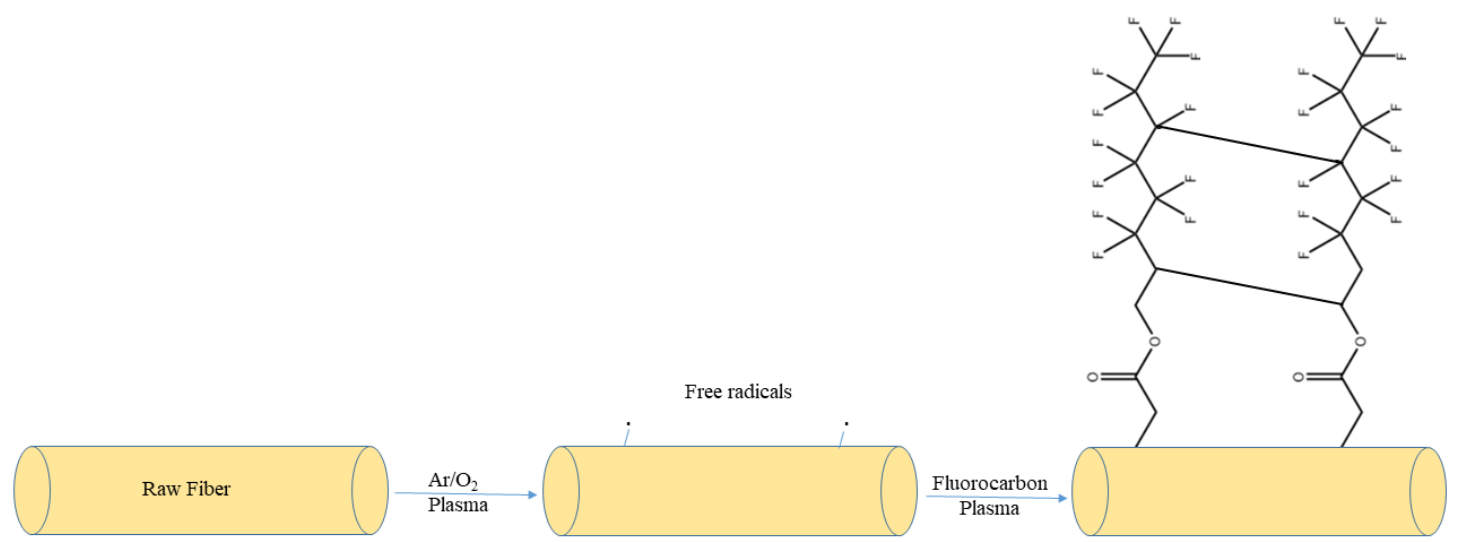

Figure 4. The schematic presentation of plasma polymerization of $1 \mathrm{H}, 1 \mathrm{H}, 2 \mathrm{H}, 2 \mathrm{H}-$ Perfluorooctyl acrylate of the fabric surface [12]

\subsection{Physical properties}

Table 2 shows the bending lengths of different samples. It can be seen that both water repellent samples showed higher bending lengths compared with the raw sample, which means an increase in the stiffness of the fabric. However, the bending length of the sample prepared by plasma coating is lower than the commercially prepared sample. This means that a better handle can be obtained when plasma technology is used for water repellent finishing of PET/Wool fabric.

The air permeability values of the samples are shown in table 3. It can be seen that the air permeability of the plasma-treated sample is near to the raw sample which confirms that a thin film of the fluorocarbon polymer has been deposited on the surface of the fibres which does block the air passing between the fibres and does not affect the air permeability and breathability of the fabric. As can be seen, the commercial process highly affected the air permeability due to binding the fibres with the high amount of the resin.

Table 2. bending lengths of different samples

\begin{tabular}{|c|c|c|}
\hline Sample & Bending length (cm) & CV\% \\
\hline Raw & 1.76 & 0.152 \\
\hline Plasma-treated & 2.23 & 0.152 \\
\hline Commercially prepared & 2.83 & 0.254 \\
\hline
\end{tabular}

Table 3. Air permeability values of different samples

\begin{tabular}{|c|c|}
\hline Sample & Air permeability $\left(\mathbf{c c} / \mathbf{s . c m} \mathbf{c m}^{\mathbf{2}}\right)$ \\
\hline Raw & 100 \\
\hline Plasma-treated & 95 \\
\hline Commercially prepared & 64.5 \\
\hline
\end{tabular}

Table 4 shows the breaking force and extension at breaking point. Both treated samples showed higher breaking force and extension compared with the raw sample. It seems that the presence of the fluoropolymer film on the surface of the fabrics increased their strength. Also, the absence of bonding between the fibres and yarns in the plasma-treated sample let the fibres to slip more easily, causing higher extension for this sample.

Table 4. Breaking force and extension at breaking point of different samples

\begin{tabular}{|c|c|c|c|c|}
\hline Sample & Extension at break (\%) & CV \% & Force (N) & CV \% \\
\hline Raw & 36.15 & 4.81 & 325 & 6.78 \\
\hline Plasma-treated & 38.33 & 2.31 & 339 & 5.60 \\
\hline Commercially prepared & 36.40 & 5.81 & 347 & 8.12 \\
\hline
\end{tabular}




\section{CONCLUSIONS}

PET/Wool fabric was subjected to water repellent finishing by two different methods including a conventional pad-dry-cure procedure by a commercial water repellent agent and plasma polymerization of $1 \mathrm{H}, 1 \mathrm{H}, 2 \mathrm{H}, 2 \mathrm{H}$-Perfluorooctyl acrylate as a new method. The results showed that the plasma treatment method results comparable results in term of water contact angle with the conventional method. However, the wash fastness of the plasma-treated sample was higher compared with the sample prepared by the conventional method.

The coating of the plasma-treated sample was more uniform and the produced film was thin and the fibres were not sticked together. This resulted in better handle and higher air permeability and extension at break for this sample. The breaking force of both samples was higher than the raw sample. The breaking force of the commercially prepared sample was a little higher than the plasma-treated sample.

The results of this study show the great potential of plasma technology for ecofriendly, uniform and long-lasting water repellent finishing of PET/Wool fabrics with minimum effect on their desirable properties.

\section{REFERENCES}

[1] Williams, J.T., Waterproof and Water Repellent Textiles and Clothing, Woodhead Publishing, Kidlington, UK, 2018

[2] Rabia, S., Muhammad, M., Naveed, R., Waqas, A.S., Qutab, H.G., Development of free fluorine and formaldehyde oil and water repellent finishes for cotton fabrics through polymerization of biobased stearic acid with carboxylic acids, In: Industria Textila, 2020, 71, 2, 145-155, http://doi.org/10.35530/IT.071.02.1731

[3] Leroux, F., Campagne, C., Perwuelz, A., Gengembre, L., Fluorocarbon nano-coating of polyester fabrics by atmospheric air plasma with aerosol, In: Appl. Surf. Sci., 2008, 254, 3902-3908

[4] Malshe, P., Mazloumpour, M., El-Shafei, A., Hauser, P., Multi-functional military textile: Plasmainduced graft polymerization of a C6 fluorocarbon for repellent treatment on nylon-cotton blend fabric, In: Surf. Coat. Technol., 2013, 217, 112-118

[5] Ceria, A., Hauser, P.J., Atmospheric plasma treatment to improve durability of a water and oil repellent finishing for acrylic fabrics, In: Surf. Coat. Technol., 2010, 204, 1535-1541

[6] Bae, G.Y., Min, B.G., Jeong, Y.G., Lee, S.C., Jang, J.H., Koo, G.H., Superhydrophobicity of cotton fabrics treated with silica nanoparticles and water-repellent agent, In: J. Colloid Interface Sci., 200, 337, 170-175

[7] Kumar, V., Pulpytel, J., Arefi-Khonsari, F., Fluorocarbon Coatings Via Plasma Enhanced Chemical Vapor Deposition of $1 \mathrm{H}, 1 \mathrm{H}, 2 \mathrm{H}, 2 \mathrm{H}$-perfluorodecyl Acrylate-1, Spectroscopic Characterization by FT-IR and XPS, In: Plasma Processes Polym., 2010, 7, 939-950

[8] Zanini, S., Freti, S., Citterio, A., Riccardi, C., Characterization of hydro- and oleo-repellent pure cashmere and wool/nylon textiles obtained by atmospheric pressure plasma pre-treatment and coating with a fluorocarbon resin, In: Surf. Coat. Technol., 2016, 292, 155-160

[9] Ramamoorthy, A., El-Shafei, A., Hauser, P., Plasma induced graft polymerization of C6 fluorocarbons on cotton fabrics for sustainable finishing applications, In: Plasma Processes Polym., 2013, 10, 430-443

[10] Wiener, J., Dejlová, P., Wicking and wetting in textiles, In: Autex Res. J., 2003, 3, 64-71

[11] Rani, K.V., Chandwani, N., Kikani, P., Nema, S.K., Sarma, A.K., Sarma, B., Optimization and surface modification of silk fabric using DBD air plasma for improving wicking properties, In: J. Text. Inst., 2018, 109, 368-375

[12] Bhatt, S., Pulpytel, J., Arefi-Khonsari, F., Low and atmospheric plasma polymerisation of nanocoatings for bio-applications, In: Surface Innovations, 2015, 3, 63-83 